Service social

\title{
Le profil de trois populations de femmes violentées sexuellement pendant l'enfance et suivies en intervention individuelle et de groupe
}

\section{Dominique Damant}

Volume 45, numéro 1, 1996

Le sexuel et le relationnel

URI : https://id.erudit.org/iderudit/706717ar

DOI : https://doi.org/10.7202/706717ar

Aller au sommaire du numéro

Éditeur(s)

École de service social de l'Université Laval

ISSN

1708-1734 (numérique)

Découvrir la revue

Citer cet article

Damant, D. (1996). Le profil de trois populations de femmes violentées sexuellement pendant l'enfance et suivies en intervention individuelle et de groupe. Service social, 45(1), 97-120. https://doi.org/10.7202/706717ar
Résumé de l'article

L'article décrit les caractéristiques d'une population de 61 femmes en intervention individuelle et en intervention de groupe au sujet d'une agression sexuelle qu'elles ont vécue pendant l'enfance. Les données sur leurs caractéristiques socio-démographiques et psycho-sociales, de même que sur les abus qu'elles ont subis, permettent de tirer des pistes de réflexion tant pour la recherche que pour la pratique. 


\title{
Le profil de trois populations de femmes violentées sexuellement pendant l'enfance et suivies en intervention individuelle et de groupe
}

\author{
Dominique DAMANT \\ Travailleuse sociale \\ Coordonnatrice de l'équipe de programmation \\ de recherche en violence conjugale du CRI-VIFF \\ (Centre de recherche interdisciplinaire sur la violence \\ familiale et la violence faite aux femmes)
}

Les recherches récentes sur les abus sexuels (Russell, 1986; Bagley 1992 ; Peters, 1988; Murphy et al., 1988; Briere et Runtz, 1987, 1988; Bagley et Young, 1990; Greenwald et al., 1990; Yama et al. 1993; Beitchman et al., 1992; Wyatt et al., 1992; Santello et Leitenberg, 1993 ; Valentine et Feinauer 1993 ; Kendall-Tackett et al., 1993) ont identifié une multitude de conséquences à long terme pour lesquelles les femmes se présentent en consultation à l'âge adulte. De façon paradoxale, la demande typique de service ne concerne pas directement l'abus, mais plutôt une ou plusieurs de ses multiples séquelles. 
Jusqu'à très récemment la majorité des professionnels ne faisaient pas de lien entre les symptômes présentés par bon nombre de victimes et l'abus vécu pendant l'enfance. Toutefois, depuis une dizaine d'années, des intervenants sociaux ont élaboré des programmes d'intervention individuelle et de groupe auprès des femmes adultes victimes d'abus sexuel pendant leur enfance.

Au Québec, les intervenants et les intervenantes qui travaillent auprès des femmes victimes d'abus sexuels pendant l'enfance s'identifient plus spécifiquement soit à l'approche systémique, soit à l'approche féministe. Cette prise de position claire quant à leur orientation théorique s'explique par des facteurs historiques. L'intérêt pour les abus sexuels découle de deux champs d'étude et d'intervention différents: le domaine de la protection de l'enfance et celui de la violence faite aux femmes.

Au Québec, les interventions en protection de la jeunesse se font dans les Centres de protection de l'enfance et de la jeunesse. L'intervention systémique a fortement influencé le travail dans les Centres de services sociaux au cours des vingt dernières années. L'importance consacrée à cette approche se comprend par le parti pris d'intervenir auprès de toute la famille et de préférer une approche thérapeutique plutôt que judiciaire. Le travail auprès des familles incestueuses a longtemps privilégié cette approche (David, 1987). L'approche de Giarretto (Giarretto, 1982), introduite au Québec vers l'année 1984, approche intégrant à la fois l'intervention judiciaire et thérapeutique, a toutefois rapidement remplacé l'unique approche systémique.

Par ailleurs, depuis le début des années 70, le féminisme a pris une place importante dans l'analyse des problème sociaux au Québec. Tout d'abord reliées aux luttes pour l'avortement libre et gratuit, les interventions féministes se sont développées dans les Centres de santé des femmes. La thérapie féministe s'est par la suite intéressée aux problèmes de santé mentale des femmes. L'expression «intervention féministe » a remplacé celle de «thérapie féministe» qui a des connotations médicales. L'intervention féministe a également pénétré les Centres locaux de services communautaires (De Koninck et Savard, 1992) à partir du développement d'interventions auprès de femmes victimes de violence conjugale (Rinfret-Raynor et al., 1989). Aujourd'hui, au Québec, la pratique féministe est très répandue tant en milieu institutionnel qu'en milieu communautaire (Bourgon et Corbeil, 1990).

Le programme le plus connu s'identifiant à l'approche Giarretto est celui offert par l'organisme Parents-Unis Lanaudière. Cette association d'entraide pour les victimes d'abus sexuels ou 
d'inceste ainsi que pour leurs familles a été fondée en 1983. Dans ses documents, Parents-Unis se donne pour mission principale de contrer la violence sexuelle de nature abusive et incestueuse à l'égard des enfants au moyen de l'entraide, de la prévention et du traitement. Le groupe de traitement où participaient les clientes décrites ici est animé par des intervenants professionnels du Centre de services sociaux, maintenant le Centre de protection de l'enfance et de la jeunesse (CPEJ) Laurentides-Lanaudière aux succursales de Repentigny et de Saint-Jérôme.

L'intervention féministe vise à replacer le problème des abus sexuels dans son contexte social. Ce modèle diffère donc de celui de Giarretto par son analyse de la problématique et il doit déboucher sur une étude plus globale des abus sexuels. Ce modèle s'adresse uniquement aux femmes victimes, et les intervenantes sont de sexe féminin. Les programmes sont donnés au CLSC Basse-Ville de Québec et dans divers Centres d'aide et de lutte contre les agressions à caractère sexuel (CALACS).

Ces deux programmes ont été décrits dans un article précédent (Damant, 1993). Par ailleurs, certaines femmes sont suivies en intervention individuelle dans des établissements du réseau, dans des organismes et par des praticiens en pratique privée. Au cours de l'automne 1992 et de l'hiver et du printemps 1993, 61 femmes molestées sexuellement pendant l'enfance qui participaient à ces différentes interventions ont été rencontrées dans le cadre d'une évaluation de ces programmes.

Le présent article expose les caractéristiques de ces 61 femmes. Jusqu'à présent aucune recherche n'a encore décrit une telle population clinique. Nous sommes donc en mesure de nous demander qui sont ces femmes: quel type d'abus ont-elles vécu, ont-elles déjà consulté un professionnel, quel est leur état psychosocial au moment où elles entreprennent l'intervention? En somme, ce portrait peut nous donner une idée de l'état des femmes qui consultent, une fois adultes, au sujet d'un abus sexuel. Cette information peut aider à formuler des objectifs d'intervention en fonction du type d'abus qu'elles ont vécu et des problèmes psychosociaux qu'elles manifestent.

\section{Provenance de la population}

La population est constituée de 61 femmes victimes d'abus sexuel pendant leur enfance, réparties en trois sous-ensembles: 
1. 25 femmes bénéficiant de l'intervention de groupe selon le modèle de Giarretto décrit ;

2. 26 femmes bénéficiant de l'intervention de groupe selon le modèle féministe ;

et

3. 10 femmes amorçant une thérapie individuelle uniquement centrée sur ce problème.

Le tableau 1 décrit la répartition des trois sous-populations.

Tableau 1

Distribution des clientes dans les trois sous-échantillons

Modèle

Nombre

de participantes

\section{Giarretto}

Repentigny 1

8

Repentigny 2

12

Saint-Jérôme

Total

5

25

Féministe

CLSC Basse-Ville

6

CALACS Saint-Georges

6

CALACS Saint-Jérôme

8

CALACS Trois-Rivières

Total

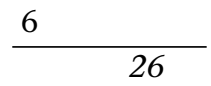

Individuel

10

TOTAL

61

Seront présentées tour à tour leurs caractéristiques sociodémographiques, la description des abus qu'elles ont subis et, enfin, l'histoire clinique des participantes.

\section{LES CARACTÉRISTIQUES SOCIODÉMOGRAPHIQUES}

Les 61 participantes à cette étude ont entre 19 et 52 ans, avec une moyenne de 34,3 ans. Les femmes les plus âgées se retrouvent 
dans le groupe de Giarretto (moyenne 33,9 ans), suivies des femmes du programme féministe (moyenne de 36,5 ans, leur âge se situant entre 22 et 52 ans). Les 10 femmes en thérapie individuelle ont en moyenne 29,4 ans et cette différence est statistiquement significative.

Les statuts civils ont été regroupés en trois catégories: célibataire, mariée ou en union de fait et séparée, divorcée ou veuve. Les célibataires sont les femmes qui n'ont jamais été mariées et qui n'ont pas de conjoint actuellement. La majorité des répondantes $(54,1 \%)$ sont mariées, $27,9 \%$ sont célibataires et $18 \%$ sont séparées, divorcées ou veuves. Près des deux tiers (64\%) des femmes inscrites en approche de Giarretto sont mariées ou en union de fait, alors que $24 \%$ sont célibataires et $12 \%$ dans la troisième catégorie. Plus de la moitié des femmes en intervention féministe vivent seules. En effet, 34,6\% sont célibataires et $23,1 \%$, séparées, divorcées ou veuves, alors que $42,3 \%$ sont mariées ou en union de fait. Enfin, $20 \%$ des femmes en thérapie individuelle seulement sont célibataires, $60 \%$ sont mariées ou vivent en union de fait et $20 \%$ sont séparées, divorcées ou veuves. Toutefois, aucune de ces différences n'est statiquement significative.

La durée moyenne de la vie de couple des femmes qui ont actuellement un conjoint est de 9 ans pour les participantes des groupes de Giarretto, 10,3 ans pour celles des groupes féministes et 8,8 ans pour celles en thérapie individuelle.

Près des trois quarts $(73,8 \%)$ des répondantes ont des enfants : $84 \%$ pour le groupe en approche de Giarretto, 73,1\% pour l'approche féministe et $50 \%$ pour la thérapie individuelle. Ces femmes ont en moyenne 1,6, 1,2 et 1 enfant respectivement. Les deux tiers $(64,4 \%)$ résident avec leurs enfants. Toutes les femmes en intervention individuelle et $81 \%$ des femmes en approche de Giarretto vivent avec leurs enfants, contre 36,8\% seulement des clientes des groupes féministes. Plusieurs répondantes n'ont pas précisé les raisons pour lesquelles leurs enfants ne cohabitaient pas avec elles. Certains enfants avaient quitté le domicile ou étaient partis étudier à l'extérieur. Quant aux jeunes enfants, ils vivaient avec leur père ou avec un autre membre de la famille ou, à quelques occasions, ils étaient placés en centre d'accueil.

Les clientes du groupe féministe sont moins scolarisées que les autres femmes, car 65,4\% d'entre elles n'ont fait que des études primaires ou secondaires contrairement à $52 \%$ et $50 \%$ 
des répondantes inscrites au programme de Giarretto et en thérapie individuelle respectivement. La majorité $(55,7 \%)$ des répondantes travaillent ( $60 \%$ pour le groupe de Giarretto et $80 \%$ pour les femmes en thérapie individuelle). Cependant, seulement 43,3\% des personnes en approche féministe travaillent.

En ce qui a trait à la description des répondantes sur le plan du statut socioéconomique, nous nous sommes ralliée à la position décrite dans l'enquête Santé Québec 1987. En effet, selon cette enquête,

la pauvreté dépasse le seul contexte du revenu monétaire pour inclure les éléments tels le patrimoine, les ressources professionnelles et scolaires, le milieu de vie, etc. ${ }^{1}$.

Il faut donc tenir compte de ces divers éléments quand on cherche à regrouper les personnes dans des catégories socioéconomiques. Quatre catégories socioéconomiques ont été développées selon l'âge des répondants et le revenu du ménage pondéré par la taille de celui-ci et la scolarité des répondants. Ces indicateurs ont l'avantage de tenir compte des aspects matériels et culturels du statut socioéconomique, en plus d'être couramment utilisés. Les quatre sous-populations ainsi formées sont: les gens très défavorisés, défavorisés, moyens et favorisés.

Comme dans l'enquête Santé Québec, les répondantes de la présente étude ont donc été classées en quatre catégories socioéconomiques. Une différence apparaît entre les femmes suivies en intervention individuelle et les femmes suivies en groupe. Bien que la majorité de toutes les répondantes rejoignent la catégorie socioéconomique moyenne $(56,5 \%, 60 \%$ et $80 \%$ pour les répondantes en approche de Giarretto, féministe et individuelle respectivement), le tiers des répondantes en approches de Giarretto et féministe se retrouvent dans les deux catégories défavorisées, alors qu'aucune des femmes en intervention individuelle ne se retrouve dans ces catégories. Près de $14 \%$ de l'ensemble de la population québécoise se répartit également dans les deux catégories défavorisées. Les personnes classées dans les deux catégories ont un revenu sous le seuil de faible revenu de Statistique Canada et une faible scolarité en fonction de leur groupe d'âge (Colin et al., 1989). Les femmes en intervention individuelle semblent un peu plus à l'aise que l'ensemble de la population québécoise, alors que les deux autres populations semblent plus pauvres. Inversement, $20 \%$ des femmes en intervention individuelle sont favorisées, contrairement à $8,7 \%$ et $4 \%$ en intervention de type Giarretto et féministe respective- 
ment. Vingt pour cent de la population québécoise se situe dans le groupe favorisé, comme les femmes en intervention individuelle. Les femmes en intervention de groupe sont quant à elles moins riches que les femmes en intervention individuelle et que l'ensemble de la population québécoise.

Le tableau 2 de la page suivante esquisse une synthèse des caractéristiques sociodémographiques des répondantes.

Des analyses statistiques ont été entreprises afin de comparer les trois groupes. Les trois sous-populations sont semblables par rapport à leur statut civil, la durée de leur vie de couple, la présence d'enfants, le nombre d'enfants, la scolarité et le travail. Par ailleurs, les femmes en intervention individuelle et féministe diffèrent quant à leur âge, les dernières étant plus âgées et ce, de façon statistiquement significative. Les femmes en intervention féministe se distinguent des autres clientes par le fait qu'elles sont moins nombreuses à avoir la garde de leurs enfants. Enfin, étant donné la très grande ressemblance entre les répondantes en intervention de Giarretto et en intervention féministe pour ce qui touche la variable socioéconomique, et la petite taille du groupe des répondantes en intervention individuelle, le $p=0,08$ nous indique une différence marquée entre les clientes en intervention individuelle et les deux autres sous-populations, les premières se situant dans les deux catégories les plus favorisées.

Les répondantes ont relaté les abus dont elles avaient été victimes dans l'enfance. Leurs caractéristiques seront décrites dans la prochaine section.

\section{LES ABUS SUBIS}

Parmi les 61 participantes qui consultent au sujet d'un abus subi pendant l'enfance, 43 ont collaboré à des entretiens téléphoniques à ce sujet, entretiens qui se sont tous déroulés après la fin de l'intervention. La présente section s'attardera à la description des abus subis. Les 43 clientes ont subi 66 abus et ce sont ces 66 abus qui seront dépeints sans tenir compte des participantes et des groupes auxquels elles appartiennent.

Les caractéristiques retenues dans la présente recherche sont: le nombre d'abuseurs, le type d'abus (inceste ou abus extrafamilial), le lien avec l'abuseur, l'âge au début de l'abus, la différence d'âge entre la victime et l'abuseur, les gestes posés par l'abuseur ou la victime, la durée de l'abus ainsi que la présence 
Tableau 2

Répartition sommaire de la population selon les caractéristiques sociodémographiques

\begin{tabular}{|c|c|c|c|c|c|c|c|c|}
\hline & \multicolumn{2}{|c|}{$\begin{array}{l}\text { Approche } \\
\text { Giarretto }\end{array}$} & \multicolumn{2}{|c|}{$\begin{array}{l}\text { Approche } \\
\text { féministe }\end{array}$} & \multicolumn{2}{|c|}{$\begin{array}{l}\text { Intervention } \\
\text { individuelle }\end{array}$} & \multicolumn{2}{|c|}{ Total } \\
\hline Âge & \multicolumn{2}{|c|}{33,9 ans } & \multicolumn{2}{|c|}{36,5 ans } & \multicolumn{2}{|c|}{29,4 ans } & \multicolumn{2}{|c|}{34,3 ans* } \\
\hline Statut civil & $\mathbf{N}$ & $\%$ & $\mathbf{N}$ & $\%$ & $\mathbf{N}$ & $\%$ & $\mathbf{N}$ & $\%$ \\
\hline Célibataire & 6 & 24,0 & 9 & 34,6 & 2 & 20,0 & 17 & 27,9 \\
\hline $\begin{array}{l}\text { Union de fait } \\
\text { ou mariée }\end{array}$ & 16 & 64,0 & 11 & 42,3 & 6 & 60,0 & 33 & 54,1 \\
\hline $\begin{array}{l}\text { Séparée, divorcée } \\
\text { ou veuve }\end{array}$ & 3 & 12,0 & 6 & 23,1 & 2 & 20,0 & 11 & 18,0 \\
\hline Total & 25 & & 26 & & 10 & & $61^{*}$ & \\
\hline \multicolumn{9}{|l|}{ Enfants } \\
\hline Pas d'enfants & 4 & 16,0 & 7 & 28,0 & 5 & 50,0 & 16 & \\
\hline 1 enfant & 7 & 28,0 & 8 & 32,0 & 1 & 10,0 & 16 & \\
\hline 2 enfants & 8 & 32,0 & 8 & 32,0 & 3 & 30,0 & 19 & \\
\hline 3 enfants & 6 & 24,0 & 2 & 8,0 & 1 & 10,0 & 9 & \\
\hline Total & 25 & & 26 & & 10 & & $61^{*}$ & \\
\hline \multicolumn{9}{|l|}{ Garde } \\
\hline Oui & 17 & 81,0 & 7 & 36,8 & 5 & 100,0 & 29 & 64,4 \\
\hline Total & 21 & & 19 & & 5 & & $45^{\star}$ & \\
\hline \multicolumn{9}{|l|}{ Scolarité } \\
\hline $\begin{array}{c}\text { Secondaire } \\
\text { et moins }\end{array}$ & 13 & 52,0 & 17 & 65,4 & 5 & 50,0 & 35 & 57,4 \\
\hline Total & 25 & & 26 & & 10 & & $61^{*}$ & \\
\hline \multicolumn{9}{|l|}{ Au travail } \\
\hline Oui & 15 & 60,0 & 11 & 43,3 & 8 & 80,0 & 34 & 55,7 \\
\hline Total & 25 & & 26 & & 10 & & $61^{*}$ & \\
\hline \multicolumn{9}{|c|}{$\begin{array}{l}\text { Catégorie socioéconomique } \\
\text { (4 catégories) }\end{array}$} \\
\hline Très défavorisée & 3 & 13,0 & 2 & 8,0 & 0 & 0,0 & 17 & 29,3 \\
\hline Défavorisée & 5 & 21,7 & 7 & 28,0 & 0 & 0,0 & 12 & 20,7 \\
\hline Moyenne & 13 & 56,5 & 15 & 60,0 & 8 & 80,0 & 36 & 62,1 \\
\hline Favorisée & 2 & 8,7 & 1 & 4,0 & 2 & 20,0 & 58 & \\
\hline \multicolumn{9}{|l|}{ ( 2 catégories) } \\
\hline Défavorisée & 8 & 34,8 & 9 & 36,0 & 0 & 0,0 & 17 & 29,3 \\
\hline Favorisée & 15 & 65,2 & 16 & 64,0 & 10 & 100,0 & 41 & 70,7 \\
\hline Total & 23 & & 25 & & 10 & & $58^{*}$ & \\
\hline
\end{tabular}

$* \leq 0,05 \quad * * \geq 0,10 \quad * * * \leq 0,01 \quad * * * \leq 0,10$


de violence ou de récompenses au moment de celui-ci. Dans les cas où les répondantes reconnaissaient avoir dévoilé l'abus, la personne à qui la victime s'est confiée et sa réaction sont retracées.

\section{LES CARACTÉRISTIQUES DE L'ABUS}

\section{Le nombre d'abuseurs}

$\mathrm{Au}$ cours de leur enfance, les répondantes ont subi de la part d'un abuseur au moins un geste sexuel non désiré. Chaque incident mettant en cause un nouvel abuseur a été considéré comme un nouvel abus. Le terme «abus» fait donc référence ici à une relation avec un abuseur, mais qui peut impliquer plus d'un incident. Près de $40 \%$ de la population étudiée a vécu des abus multiples, c'est-à-dire aux mains de plus d'un abuseur. Les femmes interrogées ont subi entre 1 et 3 abus, pour une moyenne de $1,53^{2}$. Selon plusieurs études menées auprès de populations non cliniques, la majorité des abus sont des événements uniques (Russell, 1986 ; Briere et Runtz, 1988, ; Finkelhor, 1990). La présence ici d'un si grand nombre d'abus multiples, chez des répondantes prises au sein d'une population clinique, va dans le sens des résultats des études de Briere et Runtz (1988), de Russell (1986) ainsi que de Bagley et Ramsey (1985), qui ont trouvé qu'à l'intérieur de populations non cliniques ce sont les femmes ayant subi les abus multiples qui traînent les conséquences les plus importantes.

Le tableau 3 de la page suivante résume les caractéristiques des abus subis.

\section{Le type d'abus et l'abuseur}

La grande majorité des abus sont incestueux. En effet, l'ensemble des abus $(89,4 \%$ des 66 abus subis et $90,7 \%$ des premiers abus) se retrouvent dans cette catégorie. Près de la moitié des abuseurs (43,9\% pour l'ensemble des abus et $48,8 \%$ pour le premier abus) étaient le père ou le père substitut de la victime. Viennent ensuite les cas d'inceste avec un grand-père ou un oncle $(27,3 \%$ dans l'ensemble des abus et $23,3 \%$ pour le premier abus) et les cas d'inceste avec un frère ou un cousin ${ }^{3}(18,2 \%$ pour l'ensemble des abus et $16,3 \%$ pour le premier abus). 
Tableau 3

Caractéristiques des 66 abus

\begin{tabular}{|c|c|c|c|c|c|c|}
\hline \multirow[t]{2}{*}{$\begin{array}{l}\text { Caractéristiques } \\
\text { de l'abus }\end{array}$} & \multicolumn{2}{|c|}{$\begin{array}{l}\text { Ensemble } \\
\text { des abus }\end{array}$} & \multicolumn{2}{|c|}{$\begin{array}{l}\text { Premier } \\
\text { abuseur }\end{array}$} & \multicolumn{2}{|c|}{$\begin{array}{c}\text { Autres } \\
\text { abuseurs }\end{array}$} \\
\hline & $\mathbf{N}$ & $\%$ & $\mathbf{N}$ & $\%$ & $\mathbf{N}$ & $\%$ \\
\hline \multicolumn{7}{|l|}{ Type d'abus } \\
\hline Intrafamilial & 59 & 89,4 & 38 & 88,4 & 21 & 91,3 \\
\hline Extrafamilial & 7 & 10,6 & 5 & 11,6 & 2 & 8,7 \\
\hline \multicolumn{7}{|l|}{ Abuseur } \\
\hline Père ou substitut & 29 & 43,9 & 21 & 48,8 & 8 & 34,8 \\
\hline Grand-père ou oncle & 18 & 27,3 & 10 & 23,3 & 8 & 34,8 \\
\hline Frère ou cousin & 12 & 18,2 & 7 & 16,3 & 5 & 21,7 \\
\hline Extrafamilial & 7 & 10,6 & 5 & 11,6 & 2 & 8,7 \\
\hline
\end{tabular}

Âge au début

Différence d'âge

24,8 ans

6,3 ans

Comportements abusifs

Coït ou tentative de coït

$20 \quad 31,3$

25,9 ans

21,6 ans

Contacts ou tentative

de contacts génitaux

Autres touchers

$\begin{array}{rrrrrr}38 & 59,4 & 24 & 57,1 & 14 & 63,6 \\ 6 & 9,4 & 4 & 9,5 & 2 & 9,1\end{array}$

Durée de l'abus

Courte durée

$19 \quad 30,6$

820,0

1150,0

Longue durée

4369,4

3280,0

1150,0

Présence de violence

Oui

Non

2233,3

16

37,2

626,1

$44 \quad 66,7$

27

62,8

$17 \quad 73,9$

\section{Récompenses}

Oui

Non

$14 \quad 21,2$

$12 \quad 27,9$

$2 \quad 8,7$

5278,8

$31 \quad 72,1$

2191,3

\section{Dévoilement}

Oui

Non

$34 \quad 51,5$

51,5

$24 \quad 55,8$

$10 \quad 43,5$

$32 \quad 48,5$

1944,2

1356,5

À qui

Intérieur de la famille

Extérieur de la famille

2985,3

21

87,5

$8 \quad 80,0$

$5 \quad 4,7$

3

12,5

220,0

Réaction de la personne

Comprend

$11 \quad 32,4$

$\begin{array}{ll}7 & 29,2\end{array}$

$4 \quad 40,0$

Rejette

$23 \quad 67,7$

$17 \quad 70,8$

$6 \quad 60,0$ 
Les recherches de Finkelhor (1979), Briere et Runtz (1988), Herman et al. (1986), Russell (1986) et Gomes-Schwartz et al. (1990) décrivent l'inceste père-fille comme le plus traumatisant. Le fait qu'une grande partie des clientes décrites ici aient vécu un inceste père-fille et qu'elles se retrouvent au sein d'une population clinique va dans le sens des recherches qui indiquent que les populations cliniques ont vécu les abus les plus traumatisants.

\section{L'âge au début}

Les jeunes filles avaient en moyenne 6,3 ans au moment du premier abus. Les études faites auprès de populations non cliniques rapportent des moyennes plus élevées en ce qui concerne l'âge au début de l'abus. Les femmes de la présente étude sont non seulement plus jeunes que les populations non cliniques dépeintes dans la littérature, mais également plus jeunes que les populations cliniques décrites ailleurs. Les résultats du lien entre un traumatisme et l'âge au début sont inconsistants. Il faut se garder de faire une interprétation trop hâtive des résultats présentés ici.

\section{La différence d'âge}

Pour ce qui est de la différence d'âge entre la victime et l'abuseur, elle varie entre 7 et 50 ans, avec une moyenne de 24,8 ans pour l'ensemble des abus et de 25,9 ans pour le premier abus. Étant donné cette grande différence d'âge, les résultats décrits ici semblent aller dans le sens des études de Finkelhor (1979), Briere et Runtz (1988) et Russell (1986) qui relient le traumatisme subi à la différence d'âge entre la victime et l'abuseur.

\section{Les comportements abusifs}

Les comportements abusifs ont été divisés en trois catégories: les coïts et tentatives de coït, toutes les formes de contact ou de tentative de contact génital et, enfin, les autres touchers ou tentatives de touchers. Les deux premières catégories d'abus représentent à elles seules $90,6 \%$ des abus commis $(31,3 \%$ et $59,4 \%$ respectivement pour l'ensemble des abus et $33,3 \%$ et $57,1 \%$ pour le premier abus), la dernière catégorie ne représentant que $9,4 \%$ de l'ensemble des abus commis et 9,5\% des premiers abus. Indéniablement, la population décrite ici a subi les abus rapportés dans la littérature comme les plus traumatisants. 


\section{La durée de l'abus}

Dans un premier temps, à partir des réponses obtenues au cours de l'entrevue téléphonique, les abus ont été divisés en quatre catégories: les abus uniques, les abus de courte durée (moins de cinq incidents), les incidents qui se sont produits plus de cinq fois au cours d'une seule année et les abus de longue durée, c'est-à-dire les abus qui se sont déroulés sur une période de plus d'un an et à de multiples occasions. Près de $70 \%$ de tous les abus et $80 \%$ des premiers abus se retrouvent dans les deux dernières catégories. Les résultats de la présente étude semblent aller dans le sens de la littérature qui a trouvé des corrélations positives très fortes entre un abus de longue durée et un traumatisme à l'âge adulte.

\section{La présence de violence ou de récompenses au moment de l'abus}

Les répondantes ont également été interrogées sur la présence de violence ou de récompenses au moment de l'abus. Le tiers des victimes de l'ensemble des abus $(37,2 \%$ pour le premier abus) ont subi de la violence ou des menaces au moment de l'abus. Le cinquième des femmes ont été récompensées pour les gestes abusifs. On peut imaginer l'impact que peut avoir sur un jeune enfant le fait d'être "payé » pour faire des gestes.

\section{Le dévoilement}

Enfin, 51,5\% (55,8\% au premier abus) des femmes ont dévoilé leur abus à un membre de leur famille dans $85,3 \%(87,5 \%$ des premiers abus) des cas. Parmi les réactions de la personne à qui l'abus a été dévoilé se retrouvent la négation, les punitions, les accusations, le silence, le choc et le soutien. Aux fins d'analyse, les réactions ont été divisées en deux catégories: le soutien et le rejet. Seulement $32,4 \%(29,2 \%$ au premier abus) des répondantes ont été soutenues.

Notons que l'analyse des premiers abus laisse croire qu'ils sont presque identiques à l'ensemble des abus sur toutes les dimensions, à l'exception de deux aspects: la durée de l'abus et le fait pour la victime d'être soutenue au moment du dévoilement. Le premier abus semble avoir duré plus longtemps que l'ensemble des abus ( $80 \%$ en comparaison de 69,4\%). Par ailleurs, seulement $11,5 \%$ des victimes qui ont dévoilé le premier abus ont été crues plutôt que le tiers pour l'ensemble des abus. 


\section{RÉSUMÉ}

En général, les résultats obtenus vont dans le sens des écrits sur le sujet. Les populations cliniques étudiées ici ressemblent aux populations cliniques décrites dans la littérature. Ces femmes ont subi les abus qui tendent à amener les conséquences les plus traumatisantes.

Les groupes sont comparables pour ce qui est du type d'abus subi, du nombre d'abus, de l'âge au moment du premier abus, de la différence d'âge entre la victime et l'abuseur, des comportements abusifs, de la durée de l'abus, de la violence subie au moment de l'abus, du dévoilement de l'abus et de la réaction de la personne au moment du dévoilement.

Les groupes sont différents pour ce qui est des variables «relation à l'abuseur» et "personne à qui l'abus a été dévoilé». En effet, la sous-population en approche individuelle semble avoir une plus grande prévalence de cas d'abus sexuels, alors que le groupe de Giarretto semble dévoiler plus de cas d'inceste pèrefille. De plus, les femmes du groupe féministe dévoilent moins à l'intérieur de leur famille (66,6\% en comparaison de $100 \%$ et de $83,3 \%$ ) que les femmes des deux autres groupes.

Pour terminer la description de la population à l'étude, il faut s'arrêter à l'étude de leurs traitements antérieurs et concomitants, c'est-à-dire à l'histoire clinique des participantes.

\section{L'HISTOIRE CLINIQUE DES PARTICIPANTES}

Rappelons que les clientes décrites ici se retrouvent en consultation soit en intervention individuelle seulement ou en approche de groupe selon l'approche de Giarretto ou l'approche féministe. Étant donné que l'abus qu'elles ont subi s'est déroulé au cours de leur enfance et qu'elles sont maintenant adultes, il était donc possible qu'elles aient déjà consulté un professionnel en relation d'aide soit au sujet de l'abus ou pour une autre raison. Une histoire de leurs consultations a donc été conduite, et les participantes ont été interrogées au sujet des thérapies qu'elles avaient suivies antérieurement. Le type de professionnel consulté a également été relevé ainsi que la durée moyenne de l'intervention.

Enfin, un des critères de sélection pour entreprendre les groupes selon l'approche de Giarretto et selon l'approche féministe était d'être suivie en intervention individuelle. Cet élément a également été vérifié.

Le tableau 4 fait une synthèse de cette question. 


\section{TABLEAU 4}

Répartition des répondantes selon le fait qu'elles aient consulté un professionnel en relation d'aide, le type de professionnel rencontré et la durée des rencontres

\begin{tabular}{|c|c|c|c|c|c|c|c|c|}
\hline & \multicolumn{2}{|c|}{$\begin{array}{l}\text { Groupe } \\
\text { Giarretto }\end{array}$} & \multicolumn{2}{|c|}{$\begin{array}{c}\text { Groupe } \\
\text { féministe }\end{array}$} & \multicolumn{2}{|c|}{$\begin{array}{l}\text { Intervention } \\
\text { individuelle }\end{array}$} & \multicolumn{2}{|c|}{ Total } \\
\hline & $\mathbf{N}$ & $\%$ & $\mathbf{N}$ & $\%$ & $\mathbf{N}$ & $\%$ & $\mathbf{N}$ & $\%$ \\
\hline \multicolumn{9}{|c|}{ Thérapie individuelle actuelle } \\
\hline Oui & 14 & 93,3 & 10 & 50,0 & & & 24 & 68,6 \\
\hline Non & 1 & 6,7 & 10 & 50,0 & & & 11 & $31,4^{*}$ \\
\hline \multicolumn{9}{|l|}{ Professionnel rencontré } \\
\hline Travailleur social & 9 & 64,3 & 4 & 50,0 & & & 13 & 54,2 \\
\hline Psychologue & 3 & 21,4 & 5 & 40,0 & & & 8 & 33,3 \\
\hline Autre professionnel & 2 & 14,3 & 1 & 10,0 & & & 3 & $12,5^{\star \star}$ \\
\hline \multicolumn{9}{|l|}{ Durée } \\
\hline 0-25 semaines & 3 & 21,4 & 5 & 50,0 & & & 8 & 33,3 \\
\hline 26-52 semaines & 8 & 57,1 & 2 & 20,0 & & & 10 & 41,7 \\
\hline Plus d'un an & 3 & 21,4 & 3 & 30,0 & & & 6 & 25,0 \\
\hline Total & & & & & & & $24^{\star \star}$ & \\
\hline \multicolumn{9}{|l|}{ Thérapie sur l'abus } \\
\hline Oui & 4 & 26,7 & 5 & 25,0 & 2 & 25,0 & 11 & 25,6 \\
\hline Non & 11 & 73,3 & 15 & 75,0 & 6 & 75,0 & 32 & $74,4^{\star \star}$ \\
\hline \multicolumn{9}{|l|}{ Professionnel rencontré } \\
\hline Travailleur social & 0 & 0,0 & 2 & 40,0 & 0 & 0,0 & 2 & 18,2 \\
\hline Psychologue & 0 & 0,0 & 1 & 20,0 & 2 & 100,0 & 3 & 27,3 \\
\hline Autre & 4 & 100,0 & 2 & 40,0 & 0 & 0,0 & 6 & $54,6^{\star * *}$ \\
\hline \multicolumn{9}{|l|}{ Durée } \\
\hline 8 semaines et moins & 2 & 50,0 & 3 & 60,0 & 1 & 50,0 & 6 & 54,6 \\
\hline Plus de 9 semaines & 2 & 50,0 & 2 & 40,0 & 1 & 50,0 & 5 & 45,5 \\
\hline Total & 4 & 5 & 2 & $11^{* *}$ & & & & \\
\hline \multicolumn{9}{|l|}{ Thérapie antérieure } \\
\hline Oui & 10 & 66,7 & 14 & 70,0 & 2 & 25,0 & 26 & 60,5 \\
\hline Non & 5 & 33,3 & 6 & 30,0 & 6 & 75,0 & 17 & $39,5^{\star * * *}$ \\
\hline \multicolumn{9}{|l|}{ Professionnel rencontré } \\
\hline Travailleur social & 2 & 20,0 & 2 & 14,3 & 0 & 0,0 & 4 & 15,4 \\
\hline Psychologue & 5 & 50,0 & 4 & 28,6 & 1 & 50,0 & 10 & 38,5 \\
\hline Autres professionnels & s 3 & 30,0 & 8 & 57,1 & 1 & 50,0 & 12 & $46,2^{\star *}$ \\
\hline \multicolumn{9}{|c|}{ Durée } \\
\hline 0-25 semaines & 4 & 40,0 & 6 & 42,9 & 1 & 50,0 & 11 & 42,3 \\
\hline 26-52 semaines & 3 & 30,0 & 3 & 21,4 & 1 & 50,0 & 7 & 26,9 \\
\hline Plus d'un an & 3 & 30,0 & 5 & 35,7 & 0 & 0,0 & 8 & 30,8 \\
\hline Total & 10 & & 14 & & 2 & & 26 & \\
\hline
\end{tabular}




\section{La thérapie individuelle}

Alors que 93,3\% des clientes de l'approche de Giarretto sont suivies en thérapie individuelle au moment où elles entreprennent l'intervention de groupe, seulement $50 \%$ des clientes en intervention féministe le sont. Quelle que soit l'approche, les clientes rencontrent surtout des travailleurs sociaux $(64,3 \%$ approche de Giarretto; $50 \%$ approche féministe) et la très grande majorité des répondantes (75\%) sont en thérapie depuis moins d'un an.

\section{La thérapie au sujet de l'abus}

Environ le quart des femmes ont déjà suivi une thérapie à l'âge adulte au sujet de l'abus, mais le type de professionnel consulté varie d'un groupe à l'autre. En effet, les quatre femmes en approche de Giarretto ont consulté des thérapeutes dans des groupes communautaires ou sans formation professionnelle précise, alors que les deux femmes en intervention individuelle ont consulté des psychologues. Enfin, les femmes en intervention féministe ont consulté des travailleurs sociaux (2), des psychologues (1) et des psychiatres (2). Les trois populations se répartissent également entre des thérapies à court terme (moins de huit semaines) et des thérapies de plus de neuf semaines (maximum de 103 semaines).

\section{L'autre thérapie}

Seulement le quart des femmes en thérapie individuelle ont suivi une autre thérapie, en comparaison de $66,7 \%$ des femmes en approche de Giarretto et de $70 \%$ des femmes en approche féministe. Les femmes en intervention de type Giarretto ont surtout consulté des psychologues (50\%), alors que les femmes en intervention féministe ont rencontré principalement des psychiatres et des médecins de famille $(57,1 \%)$. Les femmes en intervention individuelle ont consulté une psychologue et un psychiatre.

Peut-être parce qu'elles sont plus jeunes ou encore parce qu'elles sont moins affectées par l'abus qu'elles ont vécu, les femmes en intervention individuelle ont peu consulté jusqu'à présent. Deux d'entre elles ont consulté un psychologue au sujet de l'abus et deux ont également consulté, pour une autre raison, psychologue et psychiatre. La très grande majorité des femmes suivies en approche de Giarretto suivent actuellement (93\%) ou 
ont déjà suivi une thérapie au sujet de l'abus $(26,7 \%)$ ou pour une autre raison $(66,7 \%)$. Enfin, leur thérapie individuelle présente se fait avec un travailleur social ou une travailleuse sociale, ce qui s'explique peut-être par le fait que l'organisme ParentsUnis dirige les clientes vers ce type de professionnel. Quant aux $50 \%$ des femmes en intervention féministe, elles privilégient des psychologues (50\%) et des travailleurs sociaux (40\%). Près des trois quarts $(70 \%)$ ont déjà consulté et le quart d'entre elles ont rencontré un professionnel au sujet de l'abus. Elles semblent favoriser les consultations auprès de psychiatres ou de médecins de famille.

Les groupes se ressemblent en ce qui concerne le type de thérapeute rencontré en intervention individuelle et la durée de cette intervention, le fait d'avoir consulté sur l'abus et la durée de cette consultation, les thérapies antérieures et la durée de l'intervention. Par contre, les groupes diffèrent en ce qui a trait à la thérapie individuelle suivie parallèlement au groupe et au type de professionnel consulté antérieurement au sujet de l'abus.

Enfin, les trois sous-populations ont répondu à un questionnaire qui mesurait leur estime de soi, leurs sentiments dépressifs et leur ajustement social.

\section{L'estime de soi}

L'Index d'estime de soi de Hudson (1982) est une échelle de vingt-cinq items dont la moitié est formulée positivement, l'autre négativement. Chaque réponse est pondérée sur une échelle de cinq points entre (1) jamais et (5) toujours. Plus le score est élevé, plus la personne souffre de problèmes d'estime de soi. Hudson (1982) suggère un point de coupure de $30( \pm 5)$, les scores au-dessus de ce nombre indiquant que la personne a un problème d'estime de soi.

Les scores des 60 questionnaires retenus variaient de 6 à 86 , avec une moyenne de 47,5 . Les scores des 25 répondantes en approche de Giarretto oscillaient entre 26 et 84 (moyenne de $52,9)$. Les 26 clientes du groupe féministe avaient une moyenne de 45,8 (de 6 à 86), alors que les 9 répondantes en thérapie individuelle se situaient en moyenne à 37,6 avec des scores entre 9 et 54 . L'étude des résultats obtenus par les clientes indique que $80 \%$ de l'ensemble des trois sous-populations à l'étude manifestent des problèmes d'estime de soi ( $80 \%$ pour le groupe de Giarretto, $80,8 \%$ pour le groupe féministe et $77,8 \%$ pour les clientes en thérapie individuelle). 


\section{La dépression}

L'inventaire de dépression de Beck et al. (1961) est un instrument de vingt et une questions dont les réponses sont cotées de 0 à 3 . Plus le score est élevé, plus la personne souffre de dépression. L'inventaire mesure des symptômes précis de la dépression (poids, sommeil, pleurs, travail, etc.). Beck et al. (1988) suggère quatre points de coupure: absence de dépression ou dépression minimale: moins de 10 ; dépression légère ou modérée: de 10 à 18 ; dépression modérée à sévère: de 19 à 29 et dépression sévère: de 30 à 63 . La version québécoise de l'inventaire de la dépression de Beck (Gauthier et al., 1982) a été utilisée.

L'ensemble des scores variait entre 0 et 43 avec une moyenne de 19,69. Les 25 répondantes inscrites en approche de Giarretto avaient des scores de 2 à 41 avec une moyenne de 20,4. Le score moyen des 24 clientes des groupes féministes se situait à 22,8 , variant de 7 à 43 . Enfin, les 9 répondantes en thérapie individuelle avaient une moyenne de 9,3 et des scores de 0 à 17 . Selon les critères de Beck, les clientes en intervention individuelle ne souffriraient pas de dépression, alors que les participantes des groupes de Giarretto et féministe se situeraient dans la catégorie de dépression modérée à sévère.

\section{L'ajustement social}

L'échelle d'ajustement social (Weissman et Bothwell, 1976) contient cinquante-quatre questions mesurant la dimension affective et comportementale de l'ajustement social. Les rôles ou aspects de la vie sociale mesurés sont: l'occupation, les activités et les loisirs, les relations avec la famille élargie, le rôle conjugal, le rôle parental, l'unité familiale et, enfin, l'indépendance financière. L'instrument permet d'identifier un indice global d'ajustement qui est la moyenne de tous les items complétés, et un score moyen pour chacune des sept dimensions identifiées. Le score moyen peut varier de 1 à 5 et plus le score est élevé, plus l'ajustement est difficile.

Le score moyen des 60 questionnaires retenus est de 2,4 , les scores variant entre 1,5 et 3,9. La moyenne des scores des 25 participantes des groupes de Giarretto est de 2,5 et ils se répartissaient entre 1,5 et 3,3. Les 26 clientes des groupes féministes avaient quant à elles des scores moyens de 2,4 s'échelonnant de 1,5 à 3,9. Enfin, les 8 femmes en thérapie individuelle uniquement présentaient un score moyen d'ajustement global de 2,0 avec des variations entre 1,6 et 2,2 . 
Si l'on se base sur les recherches de Weissman et al. (1978), les scores des sous-populations suivies en approche de Giarretto et en approche féministe se rapprochent des scores des femmes souffrant de dépression sévère, alors que les scores des femmes en intervention individuelle se rapprochent des scores des femmes prises dans la population normale.

Le tableau suivant fait une synthèse des scores obtenus pour les trois sous-populations sur ces trois échelles.

\section{Tableau 5}

Synthèse des scores obtenus au prétest par l'ensemble des répondantes pour chacune des variables dépendantes

\begin{tabular}{|c|c|c|c|}
\hline & Estime de soi & Dépression & $\begin{array}{c}\text { Ajustement } \\
\text { social }\end{array}$ \\
\hline \multicolumn{4}{|c|}{ Groupe Giarretto } \\
\hline Nombre & 25 & 25 & 25 \\
\hline Moyenne & 52,9 & 20,4 & 2,5 \\
\hline Écart-type & 19,2 & 10,7 & 0,49 \\
\hline \multicolumn{4}{|c|}{ Groupe féministe } \\
\hline Nombre & 26 & 24 & 26 \\
\hline Moyenne & 45,8 & 22,8 & 2,4 \\
\hline Écart-type & 19,4 & 9,9 & 0,46 \\
\hline \multicolumn{4}{|c|}{ Intervention individuelle } \\
\hline Nombre & 9 & 9 & 9 \\
\hline Moyenne & 37,6 & 9,3 & 2,0 \\
\hline Écart-type & 13,6 & 4,8 & 0,18 \\
\hline \multicolumn{4}{|c|}{ Ensemble des répondantes } \\
\hline Nombre & 60 & 58 & 60 \\
\hline Moyenne & 47,5 & 19,7 & 2,4 \\
\hline Écart-type & 19,04 & 10,6 & 0,47 \\
\hline
\end{tabular}

Les groupes de Giarretto et féministes sont comparables pour trois variables dépendantes. Les trois groupes se ressemblent quant à la variable «estime de soi». Par ailleurs, les femmes suivies en intervention individuelle diffèrent des femmes suivies en intervention de groupe pour ce qui est des variables «dépression» et «ajustement social». 


\section{DISCUSSION}

Rappelons tout d'abord que les participantes ont vécu des agressions sexuelles beaucoup plus graves que celles vécues par les femmes prises dans des populations non cliniques. On a constaté qu'elles ont vécu à un très jeune âge

- des abus multiples;

- des incestes père-fille ;

- des comportements abusifs graves;

et

- des abus de longue durée.

Il n'est donc pas surprenant de voir ces femmes en consultation, car elles ont subi les abus qui, selon les recherches sur le sujet, comportent les éléments les plus traumatisants.

La gravité des abus subis par les répondantes a manifestement provoqué le développement d'une image corporelle négative et de sentiments négatifs à l'égard d'elles-mêmes. Plus l'abus aura été grave, plus la victime se verra de façon négative. Le maintien, à l'âge adulte, de sentiments de honte et de dégoût tels qu'établis par les instruments de mesure d'estime de soi et de dépression confirme ici les conséquences de la stigmatisation. Ce mécanisme est présent au moment de l'abus quand l'abuseur ou l'entourage de la victime lui transmet des messages négatifs à son égard, modifiant par le fait même son image de soi.

Le fait que $90 \%$ des participantes aient vécu un inceste nous permet de présumer que plusieurs d'entre elles ont vécu un sentiment de trahison, voire de perte, élément favorisant l'apparition de sentiments dépressifs. Par ailleurs, le taux élevé de dévoilements suivis d'une absence quasi unanime de soutien, laissant ainsi porter par la victime la responsabilité du geste, a pu provoquer la présence, à l'âge adulte, de sentiments de culpabilité et de responsabilité à l'égard de l'abus. Trompées par l'abuseur et abandonnées par leur milieu, il n'est pas étonnant de constater que plusieurs d'entre elles souffrent de dépression.

Le degré d'impuissance vécu au moment de l'abus a pu lui aussi induire un sentiment de perte de contrôle sur l'environnement, affectant ainsi leur estime de soi et créant ultimement des sentiments dépressifs. Chez le tiers d'entre elles qui ont vécu des abus violents et de longue durée, ces éléments n'ont pu 
qu'exacerber leur impression d'impuissance. Enfin, leur socialisation comme petite fille dans un rôle de victime a pu accentuer ce sentiment.

Le taux élevé d'incestes confirme l'existence d'une trahison. Par ailleurs, le pourcentage peu élevé de soutien au moment du dévoilement indique que les victimes, en plus d'être trahies par l'abuseur, n'ont pas été respectées par leur entourage. L'impuissance vécue dans les incestes violents et de longue durée a pu amener les victimes rendues adultes à ne pas pouvoir se défendre de façon adéquate et à revivre des relations insatisfaisantes, voire abusives. Il n'est donc pas étonnant, à la lumière des résultats obtenus ici, qu'elles aient connu des problèmes dans leurs relations interpersonnelles.

$\mathrm{Au}$ vu de ce que nous venons de présenter, on ne se surprendra pas que les victimes aient laissé voir, au moment de commencer l'intervention, des problèmes sur le plan de l'estime de soi, de la dépression et de l'ajustement social et qu'elles ressemblent en tout point aux autres populations cliniques rencontrées dans la littérature.

\section{Les femmes en intervention individuelle}

Les femmes en intervention individuelle sont plus jeunes et plus scolarisées. Elles sont plus nombreuses à travailler et à vivre dans des conditions socioéconomiques plus favorables. Le fait que ces clientes sont plus jeunes peut expliquer certaines de ces données. Ainsi, elles sont en couple depuis moins longtemps, sont moins nombreuses à avoir des enfants et en ont moins.

Elles ont vécu des abus sexuels plutôt que des incestes. Bien qu'elles manifestent des problèmes d'estime de soi et d'ajustement social, ces problèmes sont moins graves que ceux des autres sous-populations. Enfin, elles ne sont pas déprimées.

Les recherches ont montré que les incestes étaient plus traumatisants que les abus sexuels. Il serait donc permis de croire que les femmes en intervention individuelle ont été moins affectées par l'abus qu'elles ont subi que les autres répondantes. Bien que marquées par celui-ci, elles semblent avoir atteint un meilleur ajustement social, ce qui leur a peut-être permis de terminer leurs études et d'obtenir et de garder un emploi. Russell (1986) a démontré que les femmes qui avaient vécu les incestes les plus traumatisants avaient subi une baisse de mobilité sociale, telle que mesurée par une scolarité et une participation à la 
main-d'œuvre inférieures à celles de leur mère. Nous nous retrouvons possiblement devant une situation semblable. L'origine socioéconomique pourrait également influencer les conditions socioéconomiques actuelles des clientes. Une connaissance de leur milieu d'origine aurait permis d'approfondir cette question et d'identifier ce qui, de l'abus ou de l'origine sociale des répondantes, explique cette différence.

L'état psychosocial des femmes en intervention individuelle pourrait également s'expliquer par le fait qu'elles aient déjà consulté un professionnel en relation d'aide. Mais l'analyse des résultats au sujet de l'histoire clinique des participantes permet d'observer que, alors qu'environ les deux tiers des femmes en groupe ont déjà consulté, les trois quarts des femmes en intervention individuelle en sont à leur première expérience de consultation. C'est donc dire que les femmes qui ont déjà suivi des interventions sont dans un moins bon état psychosocial que les femmes qui n'ont pas encore consulté. Nous ne connaissons pas l'état des femmes qui ont déjà consulté au moment de leur première consultation. Il est d'ailleurs possible qu'au moment d'une première consultation elles aient souffert encore plus sur l'ensemble des variables. Ce résultat soulève tout de même deux questions: celui de l'inefficacité des traitements antérieurs et celui de la difficulté de traiter les conséquences à long terme de la violence sexuelle à l'égard des enfants. En effet, même si nous ne connaissons pas leur état avant leurs traitements précédents, il est indéniable que ces clientes souffrent encore, au moment de leur entrée dans les programmes de groupe, de problèmes d'estime de soi, de dépression et d'ajustement social. Ces résultats vont d'ailleurs dans la même direction que les travaux de Hughes (1990) et de Rinfret-Raynor et al. (1991), dont les populations manifestaient des problèmes d'estime de soi, de dépression ou d'ajustement social au moment d'entrer dans le programme évalué bien qu'elles aient déjà consulté. Les données recueillies ici soulignent donc leur stabilité et leur permanence.

\section{CONCLUSION}

Que pouvons-nous conclure de cette description de population clinique et quelles pistes pouvons-nous en tirer ? Tout d'abord, et il semble logique qu'il en soit ainsi, les femmes qui se retrouvent en intervention ont vécu les abus sexuels décrits dans la littérature comme les plus traumatisants. Il est donc logique que 
ces femmes se retrouvent en consultation. Ainsi, les femmes qui ont vécu des incestes semblent plus affectées que les femmes qui ont vécu un abus sexuel. Ces femmes ont également été agressées à un très jeune âge et ont subi de la violence. Lorsqu'elles ont dévoilé l'agression, elles n'ont pas été soutenues. Tous ces éléments mériteraient une analyse supplémentaire afin de déterminer lesquels de ces facteurs ont joué des rôles plus déterminants que d'autres dans le traumatisme.

Par ailleurs, une autre question est soulevée par ces résultats. Il est troublant de constater que des femmes ayant suivi de nombreuses thérapies sont encore dans un état psychosocial pour le moins difficile. Plusieurs hypothèses peuvent être développées à partir de ces données. Elles pourraient tout d'abord confirmer la permanence des conséquences des abus sexuels. Si tel est le cas des recherches doivent être entreprises afin d'identifier autant les facteurs de risque que les facteurs de protection qui permettraient aux victimes de survivre aux abus. Les résultats remettent également en question les interventions que les clientes ont suivies par le passé. Nous pouvons croire que si ni les clientes, ni les intervenants ne faisaient le lien entre le traumatisme subi et les symptômes vécus, l'intervention ne pouvait pas être efficace. Reste à savoir si le fait de suivre une intervention dont l'objectif principal est de défaire les traumatismes provoqués par l'abus sera plus efficace.

\section{Notes}

1. Colin et al., Les personnes défavorisées, Québec, Les Publications du Québec, 1989, p. 5.

2. Une seule répondante avait subi plus de trois abus. Elle a été placée dans le groupe qui avait subi trois abus.

3. Deux abus seulement mettaient en cause un cousin (deux cas de deuxième abus dans la population en approche féministe). Nous les avons joints à ceux mettant en cause des frères, étant donné la proximité sur le plan de l'âge. 


\section{Références bibliographiques}

Alexander, P.C., R.A. Neimeyer, V.M. Follette, M.K. Moore et S. HARTER (1989). "A Comparison of Group Treatments of Women Sexually Abused as Children», Journal of Consulting and Clinical Psychology, vol. 57, $\mathrm{n}^{\circ} 4$ : 479-483.

BAGLEY, C. (1992). "The Prevalence and Mental Health Sequels of Child Sexual Abuse in a Community Sample of Women Aged 18 to 27 », Canadian Journal of Community Mental Health, vol. 11 : 103-116.

BAgley, C. et R. RAMSEY (1985). «Sexual Abuse in Childhood: Psychosocial Outcomes and Implications for Social Work Practice", Journal of Social Work and Human Sexuality, vol. 4, no 1-2: 33-47.

BAGley, C. et L. Young (1990). «Depression, Self-esteem and Suicidal Behavior as Sequels of Child Sexual Abuse in Childhood: Research and Therapy», Child Maltreatment : Expanding our Concept of Helping. Hillside: Lawrence Erlbaum, 329 pages.

BeCK, A.T., R.A. STEER et M.G. GARbIN (1988). "Psychometric Qualities of the Beck Depression Inventory: Twenty-five Years of Evaluation », Clinical Psychology Review, vol. 8: 77-100.

Beck, A.T., C.H. WARD, M. Mendelson, J. Mock et J. Erbaugh (1961). "An Inventory for Measuring Depression», Archives of General Psychiatry, vol. 4 : 551-571.

Beitchman, J., K.J. Zucker, J. Hood, G.A. Da Costa, D. Akman et E. CASSAVia (1992). "A Review of Long-term Effects of Child Sexual Abuse ", Child Abuse and Neglect, vol. 16:101-118.

BRIERE, J. et M. RUNTZ (1988). "Symptomology Associated with Childhood Sexual Victimization in a Non-clinical Adult Sample», Child Abuse and Neglect, vol. 12:51-59.

Briere, J. et M. RunTz (1987). "Post Sexual Abuse Trauma », Journal of Interpersonal Violence, vol. 2, $\mathrm{n}^{\circ} 4: 367-379$.

Colin, C., J.-P. LAvoiE et C. POUlin (1989). Les personnes défavorisées. Québec: Les Publications du Québec, 119 pages.

DAVID, G. (1987). "Nécessité et efficacité d'une approche intégrée dans le traitement de l'inceste », Service social, vol. $36, n^{\text {os }} 2$ et 3 : 350-368.

FINKELHOR, D. (1979). Sexually Victimized Children. New York: Macmillan Free Press, 228 pages.

Finkelhor, D., G.T. Hotaling, I.A. LeWIS et C. SMith (1990). «Sexual Abuse in a National Survey of Adult Men and Women : Prevalence, Characteristics and Risk Factors ", Child Abuse and Neglect, vol. $14: 19-28$.

Gauthier, J., F. Thériault, C. Morin et J.S. LAWSON (1982). «Adaptation française d'une mesure d'auto-évaluation de l'intensité de la dépression », Revue québécoise de psychologie, vol. 3, $\mathrm{n}^{\circ} 2: 13-27$.

Giarretto, H. (1982). Integrated Treatment of Child Sexual Abuse: A Treatment and Training Manual. Palo Alto : Science and Behavior Books, 315 pages. 
GOMES-SCHWARTz, B., J.M. Horowitz et A.P. CARDARElli (1990). Child Sexual Abuse, the Initial Effects. Newbury Park: Sage, 205 pages.

GREenWALD, E. et H. LeITTENBERG (1990). «Posttraumatic Stress Disorder in a Non-clinical and Non-student Sample of Adult Women Sexually Abused as Children », Journal of Interpersonal Violence, vol. $5, \mathrm{n}^{\circ} 2$ : 217-228.

Greenwald, E., H. LeitTenberg, S. Cado et M.J. TARran (1990). "Childhood Sexual Abuse: Long-term Effects on Psychological and Sexual Functioning in a Non-clinical and Non-student Sample of Adult Women », Child Abuse and Neglect, vol. 14 : 503-513.

Herman, J., D. RUSSELl et K. TROCKI (1986). "Long-term Effects of Incestuous Abuse in Childhood», American Journal of Psychiatry, vol. 143, n ${ }^{\circ} 10$ : 1293-1296.

Kendall-Tackett, K.A., L. Meyer Williams et D. Finkelhor (1993). «Impact of Sexual on Children : A Review and Synthesis of Recent Empirical Studies », Psychological Bulletin, vol. 113, $\mathrm{n}^{\circ} 1$ : 164-180.

Murphy, S.M., D.G. Kilpatrick, A. AMicK-MCMullan, L.J. Veronen, J. Paduhovich, C.L. BeSt, L.A. Villeponteaux et B.E. SAUNDERS (1988). "Current Psychological Functioning of Child Sexual Assault Victims », Journal of Interpersonal Violence, vol. 3, $\mathrm{n}^{\mathrm{o}} 1$ : 55-79.

PETERS, S.D. (1984). The Relationship between Childhood Sexual Victimization and Depression among Afro-American and White Women. Los Angeles: University of California, 266 pages.

Rinfret-RAYNor, M., A. PÂQUet-DeEhy, G. LAROUChe et S. CANTIN (1989). Intervenir auprès des femmes violentées: Évaluation de l'efficacité d'un modèle féministe. Rapport de recherche $\mathrm{n}^{\circ} 1$, École de service social. Montréal: Université de Montréal, 202 pages.

Rinfret-Raynor, M., A. PÂQuet-Deehy, G. LARouche, S. CANTIN (1991). Intervenir auprès des femmes violentées : Évaluation de l'efficacité d'un modèle féministe. Rapport de recherche $\mathrm{n}^{\circ} 2$, École de service social. Montréal: Université de Montréal, 293 pages.

RuSSELL, D.H. (1986). The Secret Trauma, Incest in the Lives of Girls and Women. New York: Basic Books, 426 pages.

Santello, M.D. et H. Leitemberg (1993). "Sexual Agression by an Acquaintance: Methods of Coping and Later Psychological Adjustment», Violence and Victims, vol. 8, n ${ }^{\circ} 2: 91-104$.

VAlEntine, L. et L.L. FeinaUer (1993). "Resilience Factors Associated with Female Survivors of Childhood Sexual Abuse », The American Journal of Family Therapy, vol. 21, n $3: 216-224$.

WEISSMAN, M.M. et S. BOTHWELL (1978). "Assessment of Selfadjustment by Patient Self-report », Archives of General Psychiatry, vol. 33 : 1111-1115.

WyatT, G.E., D. GUThriE et C.M. Notgrass (1992). «Differential Effects of Women's Child Sexual Abuse and Subsequent Revictimization ", Journal of Counsulting and Clinical Psychology, vol. 60, $\mathrm{n}^{\circ} 2$ : 167-173.

YAMA, M.F., B.S. Forgas et S. TOVEY (1993). "Childhood Family Environment and Sexual Abuse as Predictors of Anxiety and Depression in Adult Women ", American Journal or Orthopsychiatry, vol. $63, \mathrm{n}^{\circ} 1: 136-141$. 